Results 116 patients (66 female, mean age 54.9 SD 17.5) have been recruited to date (55 into group 1 and 61 in group 2). In total $14(12.1 \%)$ new diagnoses of CD have been made. I-Scan appears to enhance the appearance of markers for $\mathrm{CD}$ and in a single patient in group $2 \mathrm{CD}$ markers that were not noted to be seen on WLE became apparent. Preliminary results show that endoscopic markers of $\mathrm{CD}$ across both groups currently have a sensitivity of $78.6 \%$ (48.8 - 94.3), specificity $82.4 \%$ (73.3 $88.9)$, positive and negative predictive values of $37.9 \%(21.3-$ $57.6)$ and $96.6(89.5$ - 99.1). Median tolerability scores were good in both groups but better in the I-Scan group than WLE alone (4/30 vs. $8 / 30 \mathrm{p} 0.005)$.

Conclusion The addition of I-Scan to standard endoscopy to aid the diagnosis of $\mathrm{CD}$ is well tolerated and is feasible. I-Scan appears to enhance the markings of coeliac disease, however a larger study is required to truly evaluate the effectiveness of I-Scan as an adjunct to standard endoscopy to increase CD diagnosis.

Disclosure of Interest None Declared.

\section{PTH-120 COELIAC DISEASE AND DOUBLE-BALLOON ENTEROSCOPY: WHAT CAN WE ACHIEVE? THE EXPERIENCE OF TWO EUROPEAN TERTIARY REFERRAL CENTRES}

${ }^{1} \mathrm{PD}$ Mooney*, ${ }^{2} \mathrm{C}$ Tomba, ${ }^{2} \mathrm{~F}$ Branchi, ${ }^{1} \mathrm{DS}$ Sanders, ${ }^{1} \mathrm{R}$ Sidhu, ${ }^{2} \mathrm{M}$ Locatelli, ${ }^{2} \mathrm{D}$ Conte, ${ }^{2}$ MT Bardella, ${ }^{2} \mathrm{~L}$ Elli. ${ }^{1}$ Regional GI and Liver Unit, Royal Hallamshire Hospital, Sheffield, UK; ${ }^{2}$ Center for the Prevention and Diagnosis of Celiac Disease - Gastroenterology Unit II, Fondazione IRCCS Ca' Granda Ospedale Maggiore Policlinico, Milan, Italy

\subsection{6/gutjpl-2014-307263.566}

Introduction The indications for and efficacy of device-assisted enteroscopy is not standardised in coeliac disease (CD). We present the largest study to date to evaluate the clinical role of double-balloon enteroscopy (DBE) in complicated CD.

Methods DBE findings in CD patients with suspected small bowel complications were retrospectively evaluated in two tertiary referral centres (Milan and Sheffield). Demographic data of the studied cohort were compared with a database of 1000 non complicated CD patients.

Results Findings from 14 oral and seven anal DBE in 19 CD cases (11 males $\mathrm{p}=0.003$ vs control database) were reviewed. Mean age at $\mathrm{CD}$ diagnosis $(37 \pm 19$ vs. $27 \pm 18)$ and at small bowel evaluation $(49 \pm 15$ vs. $38 \pm 13)$ was significantly higher in the DBE group compared to controls ( $\mathrm{p}$ $<0.001$ ). Indications for DBE were the follow up of known refractory coeliac disease (RCD) (\#7), suspicion of small bowel complications due to gastrointestinal symptoms (\#4), severe iron deficiency anaemia (\#6) and long standing poor dietary adherence (\#2). All DBE were performed after small bowel capsule endoscopy, except for one case. 3 patients from the known RCD group had evidence of TCRg monoclonality on biopsy (type 2 RCD). One of these patients had jejunal ulceration whilst the other 2 cases had areas with small white raised patches. A further RCD case had evidence of jejunal ulceration however biopsies didn't show any evidence of TCRg monoclonality. A single RCD case had distally worsening atrophy. Patchy small bowel atrophy was observed in all the non adherent patients and in 2 patients with persistent gastrointestinal symptoms who had only been on a gluten free diet for a short time. Two jejunal adenocarcinomas and an ileal neuroendocrine tumour were detected. All 3 of these patients presented with iron deficiency anaemia.
Conclusion This is the largest international DBE outcomes study in CD patients. Even minor mucosal lesions seen at DBE may be associated with significant pathology. Evaluation of non-responsive/refractory symptoms by DBE was associated with older patients $(p<0.001)$ and a higher proportion of males $(p=0.003)$ than an uncomplicated CD population. Further studies are needed to better understand the clinical relevance of the small bowel endoscopic features and to optimise DBE indications.

Disclosure of Interest None Declared.

\section{PTH-121 THE BURDEN OF POOR NUTRITION IN CHRONIC PANCREATITIS: WHAT ARE THE IMPACT OF BEHAVIOURAL AND SOCIOECONOMIC FACTORS?}

${ }^{1} \mathrm{~B}$ Paranandi*, ${ }^{2} \mathrm{PS}$ Patel, ${ }^{1} \mathrm{GH}$ El-Sayed, 'D Joshi, ${ }^{3} \mathrm{~A}$ Ghai, ${ }^{3} \mathrm{~K}$ Koshy, ${ }^{1} \mathrm{MH}$ Chapman, ${ }^{1}$ SP Pereira, ${ }^{1} \mathrm{GJ}$ Webster, ${ }^{1} \mathrm{GJ} J$ Johnson. ${ }^{1}$ Pancreaticobiliary Medicine, University College London Hospitals, London, UK; ${ }^{2}$ Dietetics, University College London Hospitals, London, UK; ${ }^{3}$ UCL Medical School, London, UK

\subsection{6/gutjnl-2014-307263.567}

Introduction Nutrition centred research in pancreatitis has largely focused on acute pancreatitis. There is limited nutritional data in patients with Chronic Pancreatitis (CP), a condition that predisposes to endocrine and exocrine failure, fat-soluble vitamin deficiency and osteoporosis, which may worsen a patient's quality of life and long-term outcome. We aim to determine the prevalence of malnutrition (using the Malnutrition Universal Screening Tool -MUST), active alcohol consumption and cigarette smoking in patients with $\mathrm{CP}$.

Methods Prospective study of consecutive patients with CP attending a tertiary clinic between October and December 2013. They were invited to participate in a face-to-face questionnaire study. Behavioural and socioeconomic data were collated.

Results A cohort of 86 patients identified were predominantly male (67\%), White British (62\%), median age 58 years (range 1890), of socio-economic class (SEC) 8 (21\% never worked/longterm unemployed) with educational level (EL) 1 (29\% degree or equivalent). Aetiologies included alcohol (29\%), idiopathic (25\%), autoimmune (22\%) and gallstones (11\%). The aetiology in 6\% was actively under investigation. Median follow up was 27.5 months (range $0-151$ ) from index appointment. Active alcohol exposure was noted in 33\% (28/86) with excessive amounts (m $>21 \mathrm{u}, \mathrm{f}>14 \mathrm{u} /$ week) in $8 \%(7 / 86)$. The majority of patients (> $70 \%$ ) with ongoing exposure to alcohol had primary aetiologies other than alcohol. Active cigarette smoking was noted in 34\%. MUST scores $\geq 1$ were noted in 38\% (33/86) conferring "medium to high risk of malnutrition". Multiple linear regression analysis of age, gender, ethnicity, SEC, EL, aetiology, alcohol exposure and smoking did not show any statistically significant variables that predicted MUST score. 27\% (9/33) patients "at risk of malnutrition" had been referred to or had been seen by a dietitian within the previous 12 months. The remaining 73\% (24/33) "at risk" patients all claimed to have received nil or suboptimal nutritional advice with regards to $\mathrm{CP}$ within the previous 24 months.

Conclusion Patients with CP attending our tertiary clinic are at significant risk of malnutrition, which may be under-recognised. Behavioural and socioeconomic factors do not allow reliable prediction of risk of malnutrition to be made, in this cohort. This data emphasises the need for an algorithmic approach to improve recognition of malnutrition in $\mathrm{CP}$ and for implementation of specialist dietetic, alcohol liaison and smoking cessation services within the outpatient environment.

Disclosure of Interest None Declared. 